\title{
Research on the Tower Pole Tilt Mechanism and Risk Assessment Technology Based on BeiDou reverse network RTK
}

\author{
Zhi Shen ${ }^{1}$, Wei Yu², Bao Song ${ }^{3}$, Hongwu Wang ${ }^{1}$, Junpeng $\mathrm{Li}^{1}$, Jing Jin ${ }^{1,}{ }^{*}$, Yifan Zhang ${ }^{2}$, \& Mingming Wang ${ }^{4}$ \\ ${ }^{1}$ Live work branch Company, Yunnan Power Grid Company ltd., Kunming, 650000, China \\ ${ }^{2}$ School of Remote Sensing \& Geomatics Engineering, Nanjing University of Information Science \&amp; Technology, Nanjing, 210044, \\ China \\ ${ }^{3}$ School of Geodesy and Geomatics, Anhui University of Science and Technology, Huainan, 232001, China \\ ${ }^{4}$ Jiangsu Kebo Space Information Technology Co., Ltd., Nanjing, China
}

\begin{abstract}
In order to solve the problems of inaccurate and untimely monitoring and high false alarm rate in the current monitoring of the tower pole tilt attitude, this paper proposes a technology for monitoring the tower pole tilt attitude based on RTK technology of Beidou reverse network. This method uses reverse network RTK differential positioning algorithm, which can improve the positioning accuracy of the low-cost Beidou/GNSS positioning terminal and make the positioning accuracy of the tower pole tilt monitoring reach the millimeter level. In this paper, the tilt attitude information of the transmission line tower is measured and calculated by using the height and position information of the transmission line tower, and then the risk assessment is carried out, so as to control the tilt attitude and assess the risk of the transmission line tower. The actual test results show that the method can obtain the information of the tilt attitude of transmission tower pole accurately and reliably, and can be used to evaluate it, which meets the requirements of intelligent, accurate and digital management.
\end{abstract}

\section{Introduction}

Transmission line tower, the structure of lap connecting transmission line and ground, is an important part of power system and closely related to the normal operation of power system. Due to natural environment or manmade damage, the transmission line tower pole is prone to subsidence, tilt, endanger the safety of human life and property, results the power failure $[1,2,3]$. Lots of scholars have conducted a series of studies on this issue. Some scholars studied the influence of the wind on the tower rod by using mechanical analysis and analysed the dynamic characteristics and stability of the tower rod under the action of the wind [4.5,6,7], but few did abnormal monitoring according to the structure of the tower pole. Others monitored abnormal condition of tower rod by using various sensors $[8,9,10,11,12]$, but it can only indirectly reflect the stress when the tower body has a large deformation, and can't find the load balance parameters in time, hidden faults or local member yield failure. Therefore, how to quickly and timely obtain the tilt of the tower rod and accurately get the specific position of the related tower rod has become an urgent problem to be solved in the power system. Therefore, Beidou high-precision positioning equipment can be used to build a tower pole tilt monitoring model to quickly and accurately monitor the deformation of the tower pole in real time, so as to solve the above problems [13].
In this paper, the reverse network RTK differential positioning technology will be used to improve the positioning accuracy of low-cost Beidou/GNSS positioning terminals and make the positioning accuracy of tower rod tilt monitoring reach the millimeter level, aiming to improve the level and application range of Beidou/GNSS in monitoring of tower deformation in transmission lines.

\section{RTK localization algorithm for Beidou/GNSS reverse network}

\subsection{Network RTK location algorithm}

Reverse network RTK technology (Reverse NRTK) was first proposed by Dr. Chris Rizos in 2007, and it works as shown in fig.1. Compared with the conventional RTK technology, the reverse network RTK technology can directly control and evaluate the positioning accuracy and quality of the observation data of the reference station or mobile station in the data centre, which can better ensure the accuracy and reliability of the positioning result data and realize the high-precision positioning of the singlefrequency receiver [14]. The work flow is as follows: (1) the mobile station or monitoring station transmits the observation data to the data centre through the wireless network in NMEA format; (2) the data centre obtains the

\footnotetext{
* Corresponding author: $1320013106 @$ qq.com
} 
RTCM data of the CORS data centre through the NTRIP protocol; (3) the data centre evaluates the quality of the user's observed data. If the data quality meets requirements, the user's positioning result is calculated by correcting the error related to the space distance, and the accuracy is evaluated, and the reliable positioning result is given. Otherwise, alerts are issued to the user for location or in the data center; (4) the calculated positioning results are stored in the data centre or the final user satellite information is transmitted to the user through the mobile network in the NMEA format.

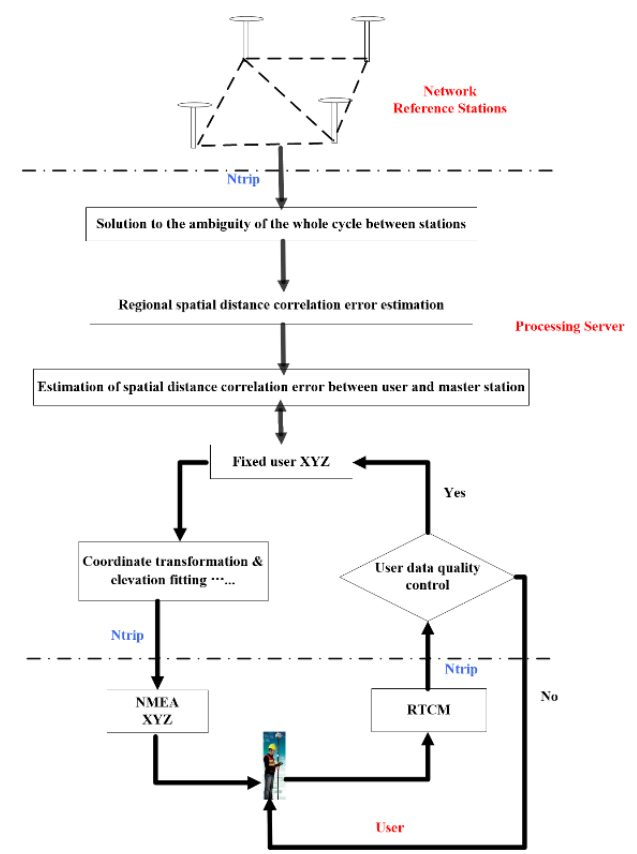

Fig. 1. Flow chart of reverse network RTK algorithm.

\subsection{RTK localization algorithm model of reverse network}

\subsubsection{Fixing the ambiguity of single epoch ultra- wide lane}

The whole week ambiguity solution between the base stations is the key to the reverse network RTK positioning, which mainly consists of the following three steps [1518] :

(1) M-W combination, formed by the carrier phase observation value and $\mathrm{p}$-code pseudo-distance, which can calculate the whole cycle ambiguity of the wide lane.

(2) Carrier phase and pseudo-distance observation values are combined with ionosphere-free, and the real solution of ionospheric fuzziness is obtained. The observation equation is as follows:

$$
\begin{aligned}
& \lambda \times \Delta \nabla \varphi_{i j}^{p q}=\Delta \nabla \rho_{i j}^{p q}-\lambda \times \Delta \nabla N_{i j}^{p q}+\Delta \nabla \operatorname{Trop}_{i j}^{p q}+\varepsilon_{i j}^{p q} \\
& \Delta \nabla P_{i j}^{p q}=\Delta \nabla \rho_{i j}^{p q}+\Delta \nabla \operatorname{Trop} p_{i j}^{p q}+\gamma_{i j}^{p q}
\end{aligned}
$$

Where $\Delta \nabla$ represents the double difference; $\lambda$ represents carrier wavelength; $\varphi$ represents observed value of the carrier; $\rho$ represents geometric distance from the station to the satellite; $N$ represents the ambiguity of the whole cycle; Trop represents tropospheric delay; $\varepsilon$ represents carrier phase observation noise; $p$ represents the observed value of pseudo-distance; $q$ represents the noise of pseudo-distance observation; $\gamma$ represents Pseudo-distance observation noise.

(3) The real solution of the narrow lane ambiguity and the corresponding covariance matrix of variance are obtained by using the solution of the wide-lane wholecycle ambiguity in step (1) and the solution of the real solution of the no-ionospheric ambiguity and the corresponding covariance matrix of variance in step (2). The whole-cycle phase fuzziness of the narrow lane is fixed by using LAMBDA algorithm, and then the wholecycle phase fuzziness of L1 and L2 is calculated.

\subsubsection{Reverse network RTK error processing}

The error of reverse network RTK mainly includes ionospheric delay, tropospheric delay and composite error including second order ionospheric delay, orbit error and multipath error. After the fixation of the whole week, the error of reverse network RTK can be calculated by using the observed values of GNSS, the double-difference fuzziness and the true coordinates of the reference station. The formula of calculation is as follows:

$$
\begin{aligned}
& \Delta \nabla \text { Ion }=\left(\frac{f_{2}^{2}}{f_{1}^{2}-f_{2}^{2}}\right)\left[\left(\lambda_{1} \Delta \nabla \phi_{1}-\lambda_{2} \Delta \nabla \phi_{2}\right)\right] \\
& +\left(\left(\lambda_{1} \Delta \nabla N_{1}-\lambda_{2} \Delta \nabla N_{2}\right)\right) \\
& \Delta \text { VTrop }=\left(M F_{w}\left(e_{m}^{k}\right)-M F_{w}\left(e_{m}^{s}\right)\right) \times Z W D_{m} \\
& -\left(M F_{w}\left(e_{n}^{k}\right)-M F_{w}\left(e_{n}^{s}\right)\right) \times Z W D_{n} \\
& +\left(M F_{H}\left(e_{m}^{k}\right)-M F_{H}\left(e_{m}^{s}\right)\right) \times Z H D_{m} \\
& -\left(M F_{H}\left(e_{m}^{k}\right)-M F_{H}\left(e_{m}^{s}\right)\right) \times Z H D_{m} \\
& \Delta \nabla \text { Other }=\Delta \nabla \rho-\left(\lambda_{1} \Delta \nabla \phi_{1}+\lambda_{1} \Delta \nabla N_{1}\right)-\Delta \nabla \text { Ion } \\
& -\Delta \nabla \operatorname{Trop}
\end{aligned}
$$

Where $\Delta \nabla$ Ion is the ionospheric delay; $f_{1}, f_{2}$ are frequency of carriers L1 and L2; $\lambda_{1}, \lambda_{2}$ are wavelength of carriers L1 and L2; $N_{1}, N_{2}$ are the full cycle ambiguity of carriers L1 and L2; $\Delta$ VTrop is tropospheric delay; MF is tropospheric mapping function; $Z W D$ is the tropospheric wet delay, which can be obtained from eq. (1). $Z H D$ is tropospheric statics delay, which can be obtained by GPT2W model. $\Delta \nabla 0$ ther is comprehensive noise; $\rho$ is the geometric distance from the station to the satellite.

After the error calculation is completed, according to the correction coordinates are provided by the mobile station, the error correction number of the mobile station can be interpolated in real time. The inverse distance weighting method is used to interpolate the above three kinds of errors of the mobile station according to the east and north direction vectors of the baseline, then to provide users with $\mathrm{cm}$ - level location services. 


\section{Principle of tower tilt measurement}

The calculation formula of the inclined angle of the tower is mainly derived from the following figure:

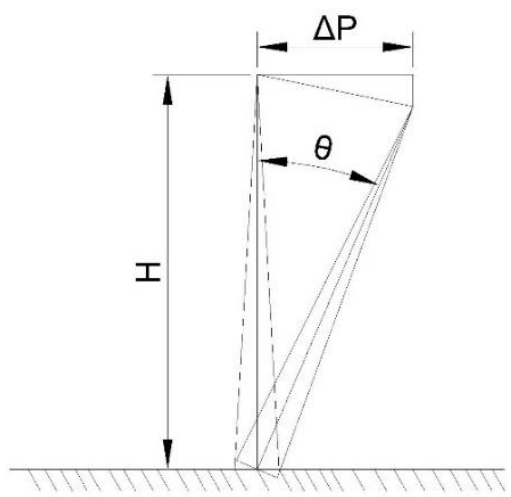

Fig. 2. The diagram among Tower Angle, tower height and plane displacement

(1) Based on Fig. 2, we can obtain calculation formula of tower tilt angle. $\left(X_{0}, Y_{0}, H_{0}\right)$ and $\left(X_{i}, Y_{i}, H_{i}\right)$ respectively represent the initial value coordinate of Beidou positioning data measurement and coordinates measured in real time.

$$
\Delta P=\sqrt{\Delta X^{2}+\Delta Y^{2}}
$$

Where $\Delta X$ and $\Delta Y$ are positioning deviation in the $\mathrm{x}$ and y directions respectively. $\Delta X=X_{i}-X_{0} ; \Delta Y=Y_{i}-$ $Y_{0}$. The root mean square error of positioning results in the $\mathrm{x}, \mathrm{y}$ and $\mathrm{p}$ directions can be calculated as follow:

$$
\left\{\begin{array}{l}
X_{R M S}=\sqrt{\frac{\sum_{i}^{n}\left(X_{i}-\bar{X}\right)^{2}}{(n-1)}} \\
Y_{R M S}=\sqrt{\frac{\sum_{i}^{n}\left(Y_{i}-\bar{Y}\right)^{2}}{(n-1)}} \\
P_{R M S}=\sqrt{\frac{\sum_{i}^{n}\left(P_{i}-\bar{P}\right)^{2}}{(n-1)}}
\end{array}\right.
$$

Where $\mathrm{n}$ represents total observations; $\bar{X} 、 \bar{Y}$ and $\bar{P}$ respectively represent the average value of the observed values in $\mathrm{x}, \mathrm{y}$ and $\mathrm{p}$ directions.

(2) The distance from the Beidou Positioning Antenna installed on the communication base station (tower) to the bottom of the tower is $H$, and the angle of the tower is $\theta$. The calculation formula of $\theta$ is:

$$
\theta=\arcsin \frac{\Delta P}{H}
$$

Where $\Delta P$ represents the plane displacement difference of tower tilt Angle; $H$ represents tower height.

\section{Results}

In the experiment, the RTK measured data at the top of the tower near Nanjing railway station was selected, and the receiver was Trimble AG332 receiver. The data were transmitted by N-trip protocol. The RTK positioning data of the research object on October 23rd, 2019 was selected, and the sampling rate was 1 second. The reference value is the average value of the whole day in the condition of no interference of the previous day, which is hereinafter referred to as the true value. Fig. 3 shows the positioning deviation results of the phase centre of the tower top receiver in the $\mathrm{x}, \mathrm{y}$ and $\mathrm{P}$ (plane) directions from the true value.

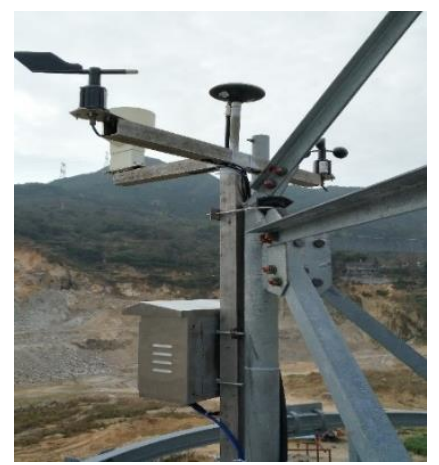

Fig. 3. Physical picture of iron tower and receiver near Nanjing railway station

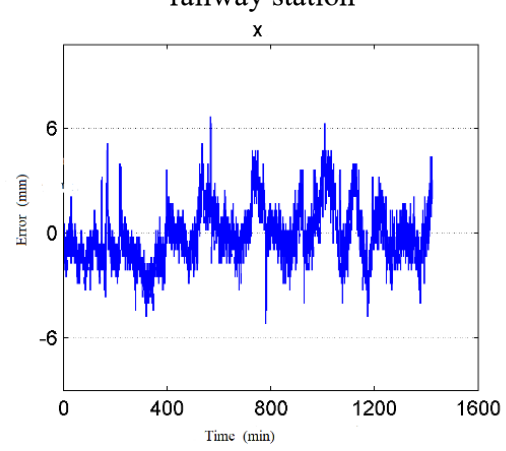

Fig. 4a. Positioning deviation in the $\mathrm{x}$ direction

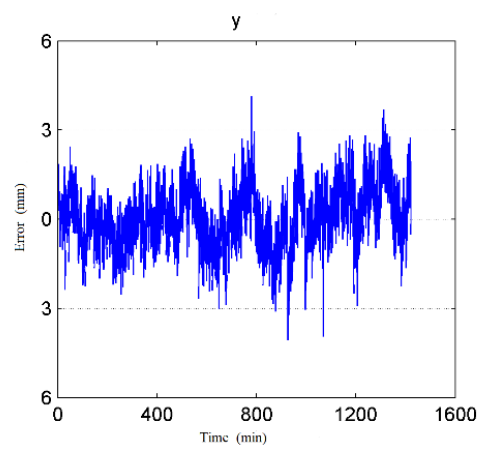

Fig. 4b. Positioning deviation in the y direction

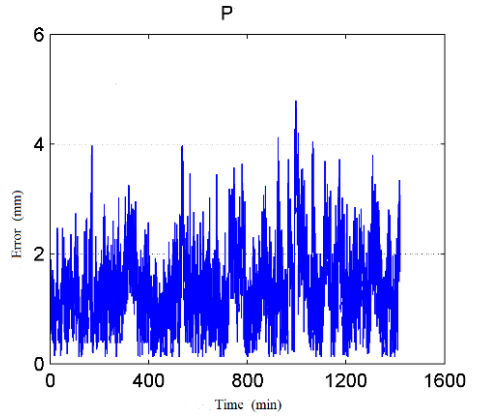

Fig. 4c. Positioning deviation in the $\mathrm{P}$ direction 
It can be seen from Fig. 4a that the positioning deviation in the $\mathrm{x}$-direction is mostly within $6 \mathrm{~mm}$, and $82 \%$ is within $5 \mathrm{~mm}$, and the median error in the $\mathrm{x}$-direction is $2.7 \mathrm{~mm}$. Fig. $4 \mathrm{~b}$ shows that most positioning deviations in the y direction are within $5 \mathrm{~mm}$, and $88 \%$ are within $3 \mathrm{~mm}$. The median error in the y direction is $1.6 \mathrm{~mm}$. Fig. 4c shows that the positioning deviation in the plane $\mathrm{P}$ direction is mostly within $4 \mathrm{~mm}$ and $75 \%$ within $3 \mathrm{~mm}$, and the median error in the P direction is $2.8 \mathrm{~mm}$, which can be considered to meet the positioning requirements of $3 \mathrm{~mm}$ level.

Fig. 5 shows the calculated tilt Angle of the tower rod at the measuring position. It can be seen that the tilt Angle of the tower rod in the above environment is between $0^{\prime} \sim 2.5^{\prime}$, with a very small fluctuation range, which meets the requirement of the power tower rod on the measuring accuracy. Moreover, the change of tower pole Angle varies with the change of time and wind environment, which has obvious space-time continuity.

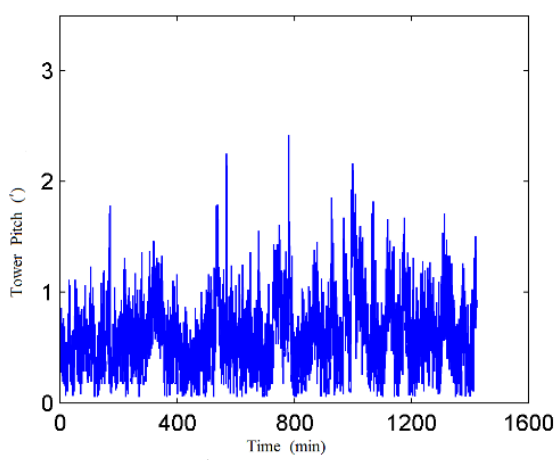

Fig. 5. The tower pole Angle at the measuring point

\section{Conclusions}

In this paper, RTK differential positioning technology of reverse network and the principle of tower pole tilt are used to analyse the measured data of RTK on the top of tower pole in a certain area. The main conclusions from the study are summarized:

(1) The coordinate component deviation of RTK differential positioning in reverse network is within $10 \mathrm{~mm}$, and $98 \%$ is within $6 \mathrm{~mm}$. The median error in $\mathrm{x}$ direction is $2.7 \mathrm{~mm}$, and the median error in y direction is $1.8 \mathrm{~mm}$. The plane positioning deviation is mostly within $4 \mathrm{~mm}$, and $75 \%$ within $3 \mathrm{~mm}$. The median error of plane $P$ direction is $2.8 \mathrm{~mm}$, which can be considered to meet the positioning requirements of $3 \mathrm{~mm}$ level

(2) Beidou tower pole tilt measurement monitoring positioning accuracy can reach the millimeter level. The tower pole tilt angle calculated after measuring is between $0^{\prime}-2.5^{\prime}$, and the fluctuation range is so small that it meets the requirement of the power tower pole for the measurement accuracy.

(3) The inclination angle of the tower pole changes with time and wind environment, and has obvious spacetime continuity.

The automatic monitoring technology of transmission line tower pole is improved by RTK of Beidou/GNSS reverse network, can effectively monitor the tilt state of the tower pole and timely carry out risk assessment, which can guarantee the continuous operation stability of the transmission line network. The experimental data show that this technology can satisfy the current stage of the transmission line tower pole intelligent, automation, digital management, has further guiding significance for the automation monitoring construction of the whole power grid system.

\section{References}

1. Wang S.L., Du Y., Sun J.X., Fang Q., Weng Y.C., Ma L., Zhang X.F., Wu J., Qin Q., Shi Q.S. Research Status of Deformation and Inclination Detection of Transmission Towers. J. Telecom Power Technology. 35:91-92(2018)

2. Meng T. Research on the Adjustment Work of Transmission Line Poles. J. Electric Engineering. 2018:122-123(2018)

3. Wu X.Q., Jiang J.Z., Li A.D., Luo Y., Huang L.H. Development and application of the tilt detection system of the split tower J. Electronics World. 2013:201(2013)

4. Zhang M., Zhao G., Wang L., Li J. Wind-Induced Coupling Vibration Effects of High-Voltage Transmission Tower-Line Systems. Shock Vib. 2017:1205976(2017)

5. Battista R.C., Rodrigues R.S., Pfeil M.S. Dynamic behavior and stability of transmission line towers under wind forces. J. Wind Eng. Ind. Aerodyn. 91:1051-1067(2003)

6. Wang Z.Q., Wang J., Huang Z.H., Wu X.Q., Meng X.B. Strain Modal Analysis of Lattice Transmission Tower. J. North China Electr. Power Univ. 44:6270(2017)

7. Wang F., Han J.K., Wang C.C., Wang C.Z., Wong X.L., Zhou Y.Y., Fang H. Strong wind simulation of transmission tower structures. Build. Struct. 48:3944(2018)

8. Xia Y., Zhang P., Ni Y.Q., Zhu H.P. Deformation monitoring of a super-tall structure using real-time strain data. Eng. Struct. 67:29-38(2014)

9. Huang X.B., Liao M.J., Xu G.H. Stress monitoring method applying FBG sensor for transmission line towers. Electr. Power Autom. Equip. 36:68-72(2016)

10. Bang H., Kim H., Lee K. Measurement of strain and bending deflection of a wind turbine tower using arrayed FBG sensors. Int. J. Prec. Eng. Manuf. 13:2121-2126(2012)

11. Zhang G.N., Design and realization of the monitoring system for the inclined angle of a kind of tower and pole J. Journal of Baoji University of Arts and Sciences(Natural Science Edition). 36:62-64(2016)

12. Hao Y.C., Su X.L., Zhao Q. E., Yan Q.X. The Design and Implementation of an Inclination Monitoring and Analysis Software for Transmission Line Tower based on Matlab J. Journal of Electric Power. 29:160164(2014) 
13. Gao P., Ji F.L., Wang J.H., Na L.D. Design of Highprecision Acquisition System for Power Grid Data Based on Beidou J. Microcomputer Applications. 35:58-61(2019)

14. Luo W.G. Analysis the Ability of Network RTK Real-time Location Service Based on BDS J. Beijing Surveying and Mapping. 33:787-791(2019)

15. Zhu H.Z., Li J., Wang C.Y., Xu A.G., Gao M. A method of BeiDou Navigation satellite system double difference network real time kinematic J. Science of Surveying and Mapping. 42:1-6+13(2017)

16. Liu M., Chai H.Z., Dong B.Q. Algorithm of Instantaneous Integer Ambiguity Resolution for Reference Stations of BDS Network RTK J. Journal of Information Engineering University. 17:760763(2016)

17. Gao W., Gao C.F., Pan S.G., Wang D.H., Wang S.L. Single-epoch Positioning Method in Network RTK With BDS Triple-frequency Widelane Combinations J. Acta Geodaetica et Cartographica Sinica. 44:641648(2015).

18. Li C.G., Luo X.J., Wang C.W., Shi X.C., Liu W.J. Preliminary Performance Exploration of Flexiblecombination BDS/GPS Network RTK Positioning J. Journal of Navigation and Positioning. 2:66-71(2014) 